\title{
The Chaos State of Internet and Coping Method
}

\author{
Xiaofeng Wang \\ Overseas Strategic Planning Division \\ Sinopec Exploration \& Production Research Institute \\ Beijing, China \\ e-mail: wangxf.syky@sinopec.com
}

\begin{abstract}
In this paper, a deterministic nonlinear model, i.e., the TCP-RED feedback system is adopted to simulate a complex computer network. The phase-space reconstruction is applied to reveal the hidden information of rate which could be obtained from the Internet easily. In addition, the maximum Lyapunov exponent is applied to judge the stability of the TCP-RED feedback system. The research demonstrates those two different parameters affect the system's stability. To avoid the system evolving into chaos state, the parameters of routers must be configured properly.
\end{abstract}

Keywords- network measurement; network stability; data center network; TCP-RED; phase-space reconstruction; maximum Lyapunov exponent

\section{INTRODUCTION}

Computer networks are complex systems. Traditionally, stochastic methods[1][2][3][4][5][6][7] are employed to analyze computer networks, however, there have been several papers which adopted deterministic nonlinear modeling in the analyses[8][9][10][11]. By virtue of nonlinearity, instability in the network may lead to oscillatory behavior. When oscillatory behavior occurs, the end-to-end jitter of the network increases, which may lead to degradation in network performance. Moreover, some networks require high quality of service (QoS) for certain applications, such as the data center network. In the data center network, the core is fully utilized. However, the longer it takes to return the instructions or data, the longer the application process remains idle, and the poorer the application performance. To avoid the delay caused by jitter, the data center network should optimally apply consistently low jitter. In this paper, we propose the model of simple computer network with Transmission Control Protocol (TCP) connections and Random Early Detection (RED) at the router end. The basic idea of TCP is to determine whether to activate the congestion control strategy and control the sender's rate. When congestion occurs, RED tries to give feedback to the sender by dropping packets. The average queue length is the parameter which determines the probability of packet loss, and several papers have discussed the instability of the average queue length [8][12][13][14]. The rate of a link is easily measured in the real network. So we apply the maximum Lyapunov exponent to estimate the instability and analyze the effect of the system parameters.

\author{
Xin Liu \\ Overseas Strategic Planning Division \\ Sinopec Exploration \& Production Research Institute \\ Beijing, China \\ e-mail: liuxin.syky@sinopec.com
}

\section{TCP-RED FEEDBACK SYSTEM MODELING BASED ON THE RATE}

In this paper, we consider a simple network of two links that are shared by many connections. As already mentioned, the network can be viewed as a network with a dominant bottleneck link and other links which are not bottlenecks. There are two routers $r_{1}$ and $r_{2}$ connected by a bottleneck link 1, and the capacity of the link is $c$. Each flow at a router sends packets with the rate of $f_{i n}^{i}$. The sending rates of all $\mathrm{n}$ flows converge at the buffer of link $l$, which generates a queue of size $q$ which is limited by its buffer size $B$. The controller at the router drops packets with a probability of $p$, which is the function of average size $q$. When the sender senses the dropping packets, it reduces the sending rate, and changes the queue size according to the controlling function, which helps to reduce the probability of packet loss.

The aim of this system is to keep the cumulative throughput lower or equal to the link's capacity c[15][16]:

$$
\sum_{i=1}^{n} r_{t, i} \leq c \quad r_{t, i}=T\left(p, R_{i}\right) \quad \mathrm{T}(p, R)=\frac{M K}{\sqrt{p} R}
$$

Where $T$ denotes Throughput of a TCP flow (in bits/sec), $M$ denotes Maximum Segment Size or Packet Size, $R$ denotes Round Trip Time, $K$ is a constant which varies between 1 and $\sqrt{8 / 3}$, and $P$ denotes the probability of packet loss.

To further simplify the system, we assume that all flows are identical in round trip time $R$, maximum segment or packet size $M$, and maximum congestion window size.

To define this control system, we model the queue as the function of control variable $q=G(p)$.

$$
q_{k+1}=G(p)= \begin{cases}\min \left(B, \frac{n K}{\sqrt{p}}-\frac{c R_{0}}{M}\right) & p \leq p_{0} \\ 0 & p>p_{0}\end{cases}
$$

Where $p_{0}=\left(\frac{n M K}{c R_{0}}\right)^{2}$ denotes the maximum probability for which the system is fully utilized, and $R_{0}$ denotes the round-trip propagation and transmission time.

According to RED, the control model can be expressed as follows: 


$$
p=H\left(\bar{q}_{k}\right)=\left\{\begin{array}{cc}
0 & 0 \leq \bar{q}_{k} \leq q_{\min } \\
\frac{\bar{q}_{k}-q_{\min }}{q_{\max }-q_{\min }} p_{\max } & q_{\min } \leq \bar{q}_{k} \leq q_{\max } \\
1 & q_{\max } \leq \bar{q}_{k} \leq B
\end{array}\right.
$$

Where $q_{\min }$ and $q_{\max }$ denote the lowest and highest threshold values respectively, and $p_{\max }$ is the selected dropping probability when $\bar{q}_{k}=q_{\max }$.

The average queue size is updated on the arrival of packets according to the exponential averaging equation:

$$
\bar{q}_{k+1}=A\left(\bar{q}_{k}, q_{k+1}\right)=(1-w) * \bar{q}_{k}+w * q_{k+1}
$$

Where $w$ is the exponential averaging weight, which determines the time constant of the averaging mechanism and how fast the RED can react to time-varying load. On one hand, the averaging weight should be selected small enough so that transient, temporary congestion does not result in an oscillation of the packet dropping probability. On the other hand, the weight should be configured large enough so that the RED can react to changes in load in a timely manner. These are conflicting goals, and the selection of the parameters affects the interaction of the RED mechanism with adaptive sources, such as TCP.

In this paper, we derive the TCP-RED feedback dynamic equation while the average queue length is the state variable:

$$
\bar{q}_{k+1}=\left\{\begin{array}{lr}
(1-w) \bar{q}_{k} & \bar{q}_{k} \geq b_{1} \\
(1-w) \bar{q}_{k}+w B & \bar{q}_{k} \\
(1-w) \bar{q}_{k}+w\left(\frac{n K}{\sqrt{\frac{p_{\max }\left(\bar{q}_{k}-q_{\min }\right)}{q_{\max }-q_{\min }}}-\frac{R_{0} c}{M}}\right) & b_{2} \leq \bar{q}_{k} \leq b_{1}
\end{array}\right.
$$

Where

And

$$
b_{1}=\left\{\begin{array}{cc}
\frac{p_{0}\left(q_{\max }-q_{\min }\right)}{p_{\max }} & p_{\max } \geq p_{0} \\
q_{\max } & p_{\max }<p_{0}
\end{array}\right.
$$

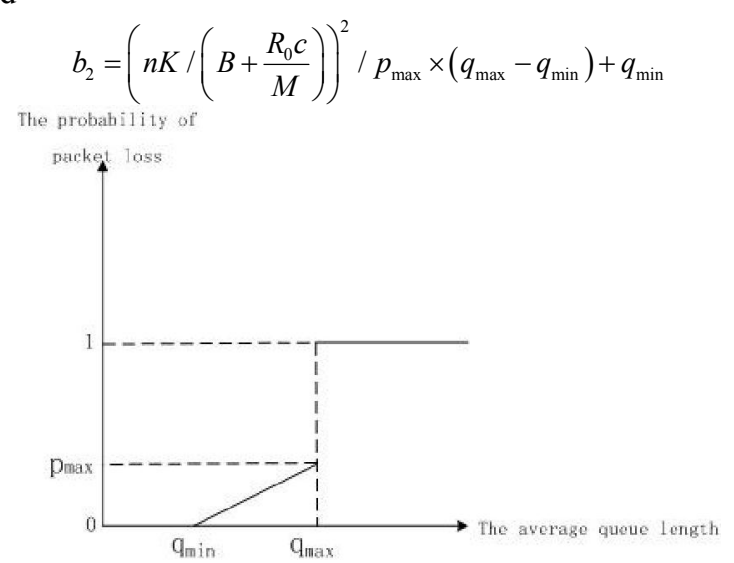

Figure 1. Relationship between the probability of packet loss and the average queue length

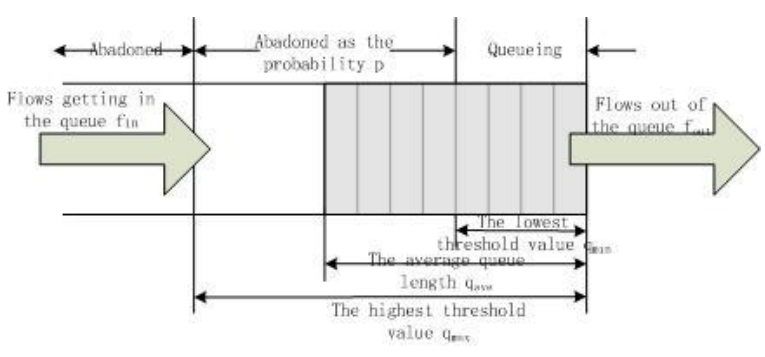

Figure 2. RED dividing the queue into three parts

In this paper, we assume that the buffer of the router is a queue, as shown in Fig.2. The equation concerning the relationship between the instantaneous queue length and the flows can be derived as:

$$
\left(f_{\text {in }}^{t}-f_{\text {out }}^{t}\right) * \Delta t=q_{t}-q_{t-\Delta t}
$$

In this paper, $f_{t}=f_{\text {in }}^{t}-f_{\text {out }}^{t}$.

According to (4) and (5), the difference between the flows and the average queue length can be derived as:

$$
f_{t+1}=\frac{\bar{q}_{t+1}}{w}+\left(1-\frac{1}{w}\right) \bar{q}_{t}-\sum_{i=1}^{t} f_{i}
$$

\section{PHASE-SPACE RECONSTRUCTION AND THE MAXIMUM LYAPUNOV EXPONENT}

\section{A. Phase-space reconstruction}

Phase-space reconstruction was first proposed by Yuie in the field of Statistics in 1927. The dynamic system has been reported in [17][18][19]. Phase-space reconstruction involves reconstructing time series from one dimension to $\mathrm{m}$ dimension, and retrieving the hidden information.

Delay-coordinate reconstruction has been extensively used. On the basis of Takens' theory, one-dimension phase space $\left\{x_{n}\right\}_{n=1}^{N}$ can be expressed as: $x_{n}=\left\{x_{n}, x_{n-\tau}, \ldots, x_{n-(m-1) \tau}\right\} \in R^{m}$, $n=N_{0}, N_{0}+1, \ldots, N$, where $x_{n}$ is the phase point for $n$, referred to as m-dimension phase space of the state. $m$ is the embedding dimension, $\tau$ is the time delay.

Therefore, it is vital to confirm the appropriate time delay and embedding dimension for phase space reconstruction, in order to fully reproduce the characteristics of the system.

\section{B. Time delay}

On the basis of Takens' theory, the time delay is selected on a random basis, under the circumstance of infinite time series and free of noise, but the opposite is the case in actual system. The basic idea of selecting the time delay is that $x_{n}$ and $x_{n+\tau}$ are independent to some degree, so it is practical to treat them as independent coordinates.

The average mutual information method [20][21] takes into account the nonlinear relation of time series, and the average mutual information $I(t)$ of time series $\left\{x_{n}\right\}_{n=1}^{N}$ between time $n$ and $n+t$ can be calculated as follows:

$$
I(t)=\sum_{n=1}^{N} P\left(x_{n}, x_{n+t}\right) \log _{2}\left[\frac{P\left(x_{n}, x_{n+t}\right)}{P\left(x_{n}\right) P\left(x_{n+t}\right)}\right]
$$


The time $\tau$ corresponds to the first local minimum of average mutual information $I(t)$, which is the time delay.

\section{Embedding dimension}

The embedding dimension $m$ is the minimum dimension which can completely contains the attractor composed by the state transition. We use the CAO method [22] to seek the important embedding dimension.

We define $R^{m}$ as the distance between $x_{i}$ and $x_{j}$, by using norms $L_{\infty}$ :

$$
\left\|x_{i}-x_{j}\right\|_{\infty}^{m}=\max _{0 \leq k \leq m-1}\left|x_{i+k \tau}-x_{j+k \tau}\right|
$$

We set $x_{r}$ as the nearest neighbor point $x_{n}$, so

$$
\begin{aligned}
R_{m}(n, r)= & \min _{j=N_{0}, \ldots, N, j \neq n}\left\|x_{n}-x_{j}\right\|_{2}^{m} \\
& =\left\|x_{i}-x_{j}\right\|_{2}^{m}
\end{aligned}
$$

In this paper, we define that $a(n, m)=\frac{R_{m+1}(n, r)}{R_{m}(n, r)}$, and the mean can be calculated as $E(m)=\frac{1}{N-N_{0}+1} \sum_{n=N_{0}}^{N} a(n, m)$.

We also define that

$$
E_{1}(m)=\frac{E(m+1)}{E(m)}
$$

In this paper, we calculate $E_{l}(m)$ as $m$ starts to increase from 1 . When $m=m_{0}$ and $E_{l}(m)$ doesn't change, we choose $m_{0}+1$ as the embedding dimension.

\section{The maximum Lyapunov exponent}

The Lyapunov exponent [23] reveals the level of convergence and divergence in the phase space neighbor track in the long term. The maximum Lyapunov exponent reflects the speed of divergence. If the maximum Lyapunov exponent is positive, the system is in an unstable state. In contrast, the system is stable if the maximum Lyapunov exponent is negative. In this paper, we use Wolf method to compute the maximum Lyapunov exponent.

We take $x_{0}$ as the initial point, whose nearest neighbor point is $\mathrm{x}_{0}$, and the distance between them is defined as $L\left(t_{0}\right)$ at moment $t_{0}$. At moment $t_{1}, x_{0}$ turns into $x_{1}$, and $x_{0}$ turns to be $x_{t 1}{ }^{\prime}$, and the distance is $L^{\prime}\left(t_{1}\right)$. Then we search the neighbor point $x_{1}$ of $x_{1}$, and calculate the distance $L\left(t_{1}\right)$ between them. We repeat the above procedure until it traverses the entire time series. The process is as shown in Fig.3.

The equation of the maximum Lyapunov exponent is as follows:

$$
\lambda_{1}=\frac{1}{t_{M}-t_{0}} \sum_{k=1}^{M} \log _{2} \frac{L^{\prime}\left(t_{k}\right)}{L\left(t_{k-1}\right)}
$$

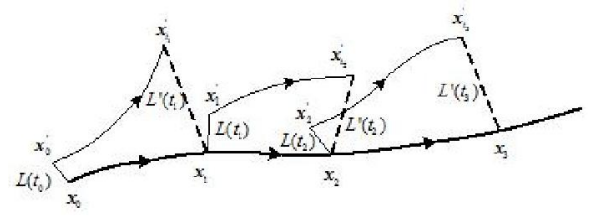

Figure 3. Procedure for calculating the maximum Lyapunov exponent

\section{NUMERICAL RESULTS AND ANALYSES}

\section{A. Parameters of phase-space reconstruction}

In this section, we use MATLAB to simulate the TCPRED feedback system and analyze the instability and the effect of system parameters. Phase-space reconstruction is an important step to retrieve the hidden information so we should choose the parameters carefully. We calculate the time delay with the average mutual information method, as shown in Fig.4, in which the time delay is $\tau=3$. In addition, we use CAO method to obtain the embedding dimension. Fig.5 indicates that $E$ reaches saturation state when the embedding dimension is equal to 3 . According to the $\mathrm{CAO}$ method, we choose 4 as the embedding dimension.

\section{B. Effect of the system parameters}

\section{1) Effect of exponential averaging weight $w$}

The following parameters are common to the next simulation.

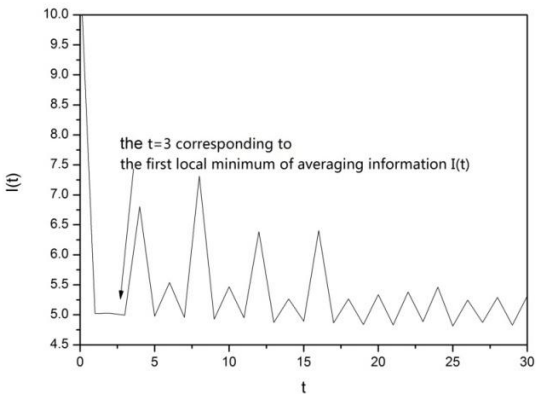

Figure 4. Time delay

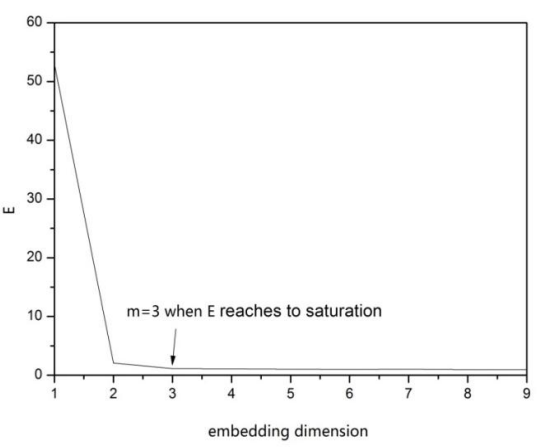

Figure 5. Embedding dimension

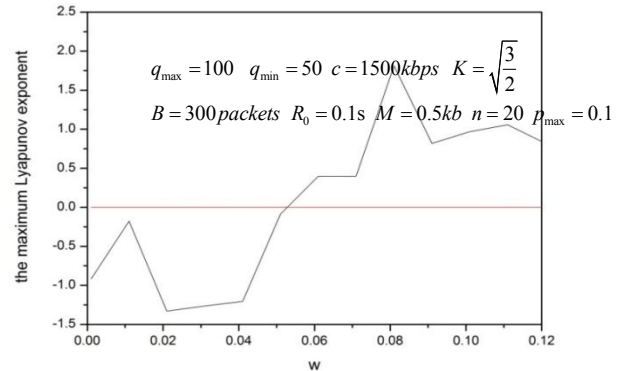

Figure 6. The maximum Lyapunov exponent of the averaging weight $w$ 


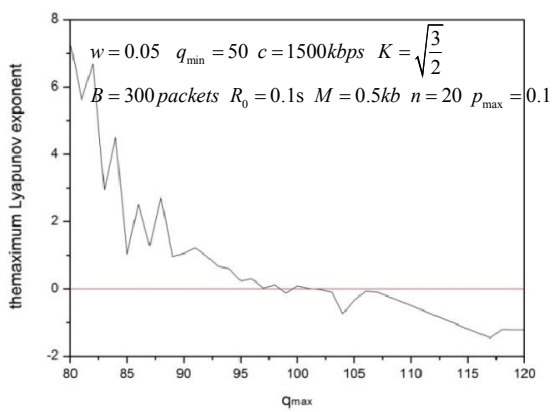

Figure 7. The maximum Lyapunov exponent of RED control parameter

Fig.6 shows that the exponent is negative at the beginning of simulation. It increases to zero and then decreases straight away while averaging weight continues to increase. Then it becomes a positive value after increasing more than a fixed point. A positive Lyapunov exponent confirms the presence of chaotic behavior. Fig. 6 implies that we should set the averaging weight less than the fixed point when we configure the routers, otherwise it will lead to chaos state.

\section{2) Effect of RED control parameter $q_{\max }$}

The following parameters are common to the next simulation.

In this sub-section, we discuss the effect of RED control parameter $q_{\max }$. Unlike the averaging weight, it remains positive at the beginning of simulation. The maximum Lyapunov exponent decreases while $q_{\max }$ increases. When $q_{\max }$ is almost equal to 100 , the maximum Lyapunov

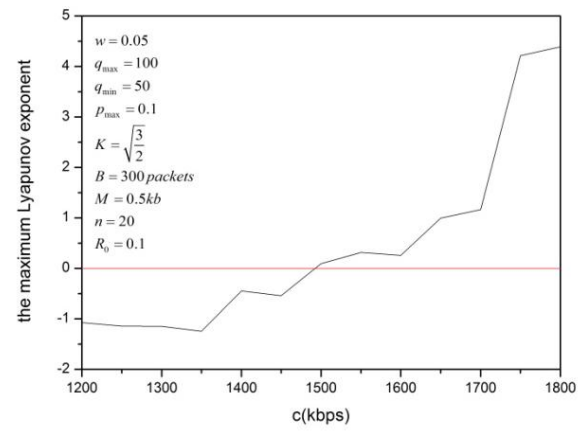

(a) The maximum Lyapunov exponent of link capacity

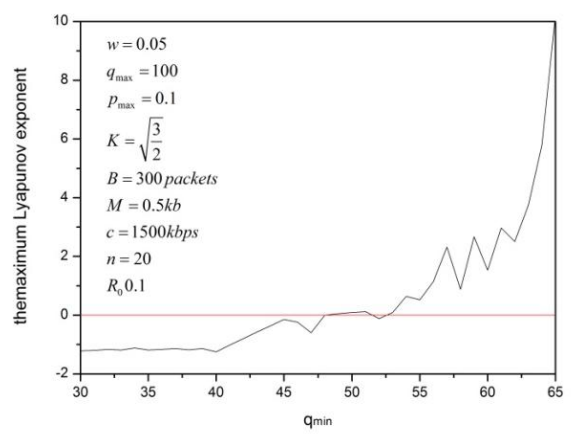

(c) The maximum Lyapunov exponent of $q_{\min }$ exponent turns to negative. Then it stays negative while $q_{\max }$ further increases. Fig.7 demonstrates that the system turns to be stable as $q_{\max }$ increases. The case is almost the opposite to the averaging weight, so we can conclude that there exist two different effects among the system parameters. Therefore, it is necessary to compute the maximum Lyapunov exponent for the rest of the system parameters.

3) Effect of system parameters on stability

To understand the effects of other system parameters on system stability, we plot the Lyapunov diagram. Other simulation parameters for this Lyapunov diagram are as follows:

As shown in Fig.8, the maximum Lyapunov exponent remains negative before the fixed point and it keeps the system stable. After the fixed point, the system becomes unstable. Therefore, when choosing the system parameters, we should set them less than the fixed point in order to keep the system stable.

\section{CONCLUSION}

In this paper, we use the maximum Lyapunov exponent to analyze the TCP-RED feedback system based on the rate, which is most easily achieved on the Internet. We simulate the maximum Lyapunov exponent of different system parameters in order to find out how these parameters affect the system stability. There are two different effects. In some cases, the system stability decreases while the parameter value increases, such as the averaging weight $w$.

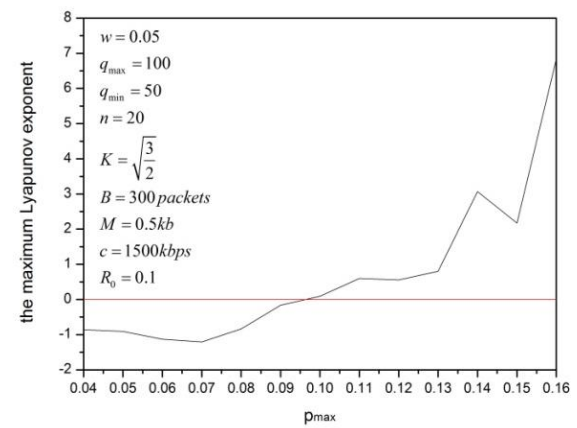

(b) The maximum Lyapunov exponent of $p_{\max }$

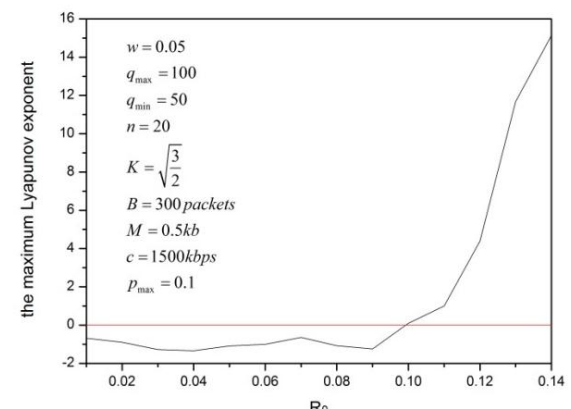

(d) The maximum Lyapunov exponent of $R_{0}$

Figure 8. The maximum Lyapunov exponent of other system parameters 
There is a fixed point to distinguish the stability and instability of system. In other cases, the system stability increases while the parameter value increases, such as the RED control parameter $q_{\max }$. There is also an apparent fixed point. Once we know the effects, when configuring the routers, we can choose the system parameters appropriately to avoid inducing system instability.

\section{ACKNOWLEDGMENT}

This work was supported by the National Science and Technology Major Project of China (2011ZX05031-001$007 \mathrm{HZ}$ ).

\section{REFERENCES}

[1] Hollot C, Misra V, Towsley D, et al. A Control Theoretic Analysis of RED.Proceedings of Twentieth Annual Joint Conference of the IEEE Computer and Communications Societies: April 2226,2001,Alaska,USA.

[2] Bonald T,May M,Bolot J-C.Analytic Evaluation of RED Perfoemance. Proceedings of 19th Annual Joint Conference of the IEEE Computer and Communications Societies:March 26-30,2000, Tei-Aviv,Israel

[3] Chen X, Wong S-C and Tse C K. Adding Randomness to Modeling Internet TCP-RED Systems With Interactive Gateways[J]. IEEE transactions on circuits and systems-ii: express briefs, vol. 57 , no. 4 , April 2010.

[4] Wong S-C., Tse C K and Lau F C M. Dynamics of Oscillatory Queue Length in TCP-RED Gateway.Proceedings of 2005 International Symposium on Intelligent Signal Processing and Communication Systems: December 13-16, 2005,Hong Kong, China.

[5] Pei L, Zhang L, Wang R. Co-dimension 1 bifurcations of the delayed Internet TCP-RED model.2010 International Conference on Multimedia Information Networking and Security: November 4 6,2010,Nanjing,China.

[6] Zhang K, Cheng P F, Man Z, et al. Queue Dynamics Analysis of TCP Veno with RED. Proceedings of Wireless Communications and Networking Conference: March 11-15,2007,Hong Kong,China.

[7] Baiocchi A and Vacirca F. TCP fluid modeling with a variable capacity bottleneck link. Proceedings of 26th Annual Joint Conference of the IEEE Computer and Communications Societies: May 6-12 2007,Alaska,USA.

[8] Firoiu V and Borden M. A Study of Active Queue Management for Congestion Control.Proceedings of 19th Annual Joint Conference of the IEEE Computer and Communications Societies: March 2630,2000, Tei-Aviv,Israel.

[9] Misra V, Gong W and Towsley D.A Study of Active Queue Management For Congestion Control. Proceedings of 19th Annual Joint Conference of the IEEE Computer and Communications Societies: March 26-30,2000,Stockholm,Sweden.

[10] Kuusela P, Lassila P and Virtamo J. Stability of TCP-RED Congestion Control. Submitted for publication, available at http://www.tct.hut..fi/tutkimus/com2/publ/,2000.

[11] Veres A and Boda M. The Chaotic Nature of TCP Congestion Control. Proceedings of 19th Annual Joint Conference of the IEEE Computer and Communications Societies: March 26-30,2000, TeiAviv,Israel.

[12] Ranjan P, Abed E.H. and La R.J. Nonlinear instabilities in TCP-RED. Proceedings of 21st Annual Joint Conference of the IEEE Computer and Communications Societies: June 23-27,2002,New York,USA.

[13] Chen W, Li Y, Yang S-H. An Average Queue Weight Parameterization in a Network Supporting TCP Flows with
RED.Proceedings of the 2007 IEEE International Conference on Networking, Sensing and Control: April 15-17 2007,London,UK.

[14] Haider A, Sirisena H, Sreeram V, et al. Stability Conditions For Scalable TCP-RED Based AQM.Proceedings of the 2007 IEEE International Conference on Mechatronics and Automation: August 5-8,2007,Harbin,China.

[15] Mathis M, Semke J, Mahdavi J,et al. The Macroscopic Behavior of the TCP Congestion Avoidance Algorithm. Computer Communication Review, Vol 27,no.3,1997.

[16] Padhye J, Firoiu V, Towsley D, et al.Modeling TCP Reno performance: a simple model and its empirical validation.IEEE/ACM Trans.Networking, vol.8, pp.133-145, Apr.2000.

[17] Packard N H, Griuthfield J P, Farmer J D, et al. Geometry from a time series. Physical Review Letters, 1980, 45(9): 712 716.

[18] Takens F. On the numerical determination of the dimension of an attractor. In Rand D, Young L S editors. Dynamical Systems and Turbulence. Warwick, 1980, Lecture Notes in Mathematics, SpringerVerlag, 1981, 898: 366 381.

[19] Mane R. On the dimension of the compact invariant sets of certain nonlinear maps. In Rand D, Young L S editors. Dynamical Systems and Turbulence. Warwick, 1980, Lecture Notes in Mathematics, Springer-Verlag, 1981, 898: 230.

[20] Fraser A M, Swinney H. Independent coordinates for strange attractors from mutual information. Physical Review A, 1986, 33(2): 1134 1140.

[21] Fraser A M. Information and entroy in strange attractors. IEEE Trans. on Information Theory, 1989 , 35(2): 245 262.

[22] Cao L Y. Practical method for determining the minimum embedding dimension of a scalar time series. Physica D, 1997, 110(1-2): 43 50.

[23] Wolf A, Swift J B, Swinney H L et al. Determining Lyapunov exponents from a time series. Physica D, 1985, 16: 285 317. 